\title{
Wnt4 overexpression promotes thymoma development through a JNK-mediated planar cell polarity-like pathway
}

\author{
YUAN CHEN, PENG ZHANG, PEIYUAN TANG, PENG LV, XIN LI, YUANGUO WANG, YANG LV and YIMEI LIU
}

Department of Cardiothoracic Surgery, Tianjin Medical University General Hospital, Tianjin 300052, P.R. China

Received December 20, 2015; Accepted June 9, 2017

DOI: $10.3892 / 01.2017 .7266$

\begin{abstract}
Thymoma is the most common neoplasm of the anterosuperior mediastinum. Activation of the Wnt signaling pathway has a role in a variety of human cancers. The present objective was to examine c-Jun $\mathrm{N}$-terminal kinase (JNK) mRNA and protein expression in thymoma cells undergoing apoptosis subsequent to downregulation of Wnt 4 . Wnt4 and JNK mRNA and protein expression was analyzed by reverse transcription-quantitative polymerase chain reaction and immunohistochemistry, respectively, in 15 thymoma tissues and 6 thymus cyst tissues. Thymoma cells were cultured and transfected with shRNA plasmids targeting the Wnt4 gene. Wnt 4 and JNK protein expression was detected by western blot analysis. Apoptosis was analyzed using Wright-Giemsa staining, Hoechst-33342/propidium iodine double staining and flow cytometry. The results showed that Wnt 4 and JNK mRNA and protein expression were significantly increased in thymoma compared with normal thymus tissue. Subsequent to transfection, thymoma Wnt4 and JNK mRNA and protein expression were significantly decreased in shRNA-treated groups, with the strongest inhibition being $52.37 \%$. Characteristic apoptotic morphological changes were observed and apoptosis increased. Overall, the present concluded that Wnt4 has an important role in thymoma development, which appears to be activated through a JNK mediated planar cell polarity-like pathway.
\end{abstract}

\section{Introduction}

Thymoma is the most common neoplasm of the anterosuperior mediastinum, accounting for $\sim 20 \%$ of all mediastinal tumors and $50 \%$ of all anterior mediastinal tumors in adults (1). Thymomas occur at all ages, but there is a broad peak between 35 and 70 years of age. Men and women are approximately equally affected, although it is slightly more common in older women (2). Numerous thymoma cases present with systemic

Correspondence to: Professor Peng Zhang, Department of Cardiothoracic Surgery, Tianjin Medical University General Hospital, 154 Anshan Street, Heping, Tianjin 300052, P.R. China E-mail: zhangpengtjgh@126.com

Key words: Wnt4, JNK, thymoma, RNAi, apoptosis syndromes, such as myasthenia gravis, or other associated syndromes, such as immune and autoimmune diseases. Surgery remains the main treatment for thymoma, as thymoma pathogenesis is poorly understood (3).

The Wnt signaling pathway is one of a handful of evolutionarily conserved signal transduction pathways that are used extensively during animal development (4). Wnt signals control multiple biological processes, including cellular proliferation, fate specification, polarity and migration. Activation of the Wnt signaling pathway plays a role in a variety of human cancers (5-7), and regulates T cell development (8). In the thymus, Wnt ligands are expressed primarily on thymus epithelial cells and activate a highly complex signaling network via G-protein dependent Frizzled receptors (9).

Wnt 4 is one of the Wnt proteins that can activate non-canonical pathways. It has an important role in thymus development and maintenance of the thymus microenvironment $(10,11)$. JNK signaling is necessary for planar cell polarity (PCP)-like pathways (12), including one of the non-canonical Wnt pathways. The present study examined the expression of Wnt4 and JNK mRNA and protein in thymoma. Wnt4 gene expression was then blocked in thymoma cells by short hairpin (sh)RNA interference, and changes in JNK mRNA and protein expression and thymoma apoptosis were then examined. The results indicate the Wnt4/JNK signaling has an important role in thymoma development and proliferation.

\section{Materials and methods}

Reverse transcription-quantitative polymerase chain reaction (RT-qPCR) analysis of thymoma Wnt4 and JNK mRNA expression. Thymoma and normal thymus tissue samples were collected from 15 patients with thymoma and 6 patients with thymus cysts at the Tianjin Medical University General Hospital (Tianjin, China). The Tianjin Medical University General Hospital Ethics Committee approved the present study and written informed consent was obtained from all patients. A definite pathological diagnosis was established in patients who underwent surgery but had not received chemotherapy or radiotherapy between January 2013 and December 2014. RNA was extracted by tissue homogenization in TRIzol (Invitrogen; Thermo Fisher Scientific, Inc., Waltham, MA, USA), according to the manufacturer's instructions. Total RNA $(1 \mu \mathrm{g})$ was reverse transcribed into cDNA and quantified by RT-qPCR using SYBR Premix Ex Taq ${ }^{\text {TM }}$ (Takara Bio, 
Inc., Otsu, Japan). PCR primer pairs for human Wnt4, JNK and GAPDH (Table I) were designed using GeneRunner (Aoke Biological Technology, LLC, Beijing, China). Amplification was performed at $94^{\circ} \mathrm{C}$ for $5 \mathrm{~min}$ for predenaturation, $94^{\circ} \mathrm{C}$ for $40 \mathrm{sec}$ for denaturation, $59^{\circ} \mathrm{C}$ for $30 \mathrm{sec}$ for annealing and $72^{\circ} \mathrm{C}$ for $30 \mathrm{sec}$ for extension, for a total of 45 cycles, with a final extension at $72^{\circ} \mathrm{C}$ for $10 \mathrm{~min}$. Wnt4 and JNK PCR products were purified by $1.2 \%$ agarose gel and the purpose strip image was taken by GelDoc 2000 gel imaging system (Bio-Rad Laboratories, Inc., Hercules, CA, USA). Positive bands were measured with gray values and sequenced. The relative expression of target genes was determined using the $2^{-\Delta \Delta \mathrm{Cq}}$ method (13).

Immunohistochemistry of Wnt4 and JNK protein expression in thymoma tissues. Thymoma tissue was fixed in $4 \%$ paraformaldehyde PBS for $12 \mathrm{~h}$ at room temperature. Following dehydration, cleared and paraffin embedded thymoma tissue sections $(4 \mu \mathrm{m})$ were deparaffinized, dehydrated and subjected to antigen retrieval with Tris-EDTA by high temperature $\left(117^{\circ} \mathrm{C}\right)$ and high pressure $(180 \mathrm{kPa})$ for $3 \mathrm{~min}$. Tissue sections were blocked using $3 \%$ hydrogen peroxide solution for $10 \mathrm{~min}$ at $37^{\circ} \mathrm{C}$ and non-specific binding was blocked using $10 \%$ normal goat serum (cat. no. SP-9000, SPlink Detection kits; ZSGB-BIO Technologies, Inc., Beijing, China) for $10 \mathrm{~min}$ at room temperature. Sections were incubated with rabbit anti-Wnt4 polyclonal antibody (dilution 1:100, cat. no. ab189037; Abcam, Cambridge, UK) and mouse anti-JNK monoclonal antibody (dilution 1:25, cat. no. ab201624; Abcam) overnight at $4^{\circ} \mathrm{C}$. The sections were incubated with $100 \mu \mathrm{l}$ goat anti-rabbit IgG secondary antibody for $30 \mathrm{~min}$ at $37^{\circ} \mathrm{C}$ (ready to use, cat. no. SP-9000; ZSGB-BIO Technologies, Inc.). Brown granules indicated expression. The frequency of positive cells in five high-power fields (magnification, x400, Leica DMIL; Leica Microsystems GmbH, Wetzlar, Germany) of each section was determined by counting at least 2,000 thymoma cells in a blinded manner.

Cell source and culture. A human thymoma cell line was derived in vitro from a 50-year old Chinese man with AB-type thymoma and myasthenia gravis. The cell line was maintained in primary culture at Tianjin Medical University General Hospital in Dulbecco's modified Eagle's medium (DMEM, Gibco; Thermo Fisher Scientific, Inc.) supplemented with $10 \%$ fetal bovine serum (Gibco; Thermo Fisher Scientific, Inc.), $100 \mathrm{IU} / \mathrm{ml}$ penicillin and $100 \mu \mathrm{g} / \mathrm{ml}$ streptomycin, at $37^{\circ} \mathrm{C}$ in a humidified $5 \% \mathrm{CO}_{2}$ atmosphere (14).

Construction and selection of interference plasmid. A Psi-H1 shRNA expression vector was chosen to interfere with the plasmid hairpin loop sequence TCAAGAG. Four Wnt4 shRNA interference plasmids were constructed using the HSHTR001 plasmid, which also acted as an empty plasmid control. All plasmids were obtained from Guangzhou Fulengen Co., Ltd. (Guangzhou, China) (Table II). Plasmid amplification and extraction used Trans1-T1 phage-resistant competent cells (Wuhan Cell Marker Biotechnology Co., Ltd., Wuhan, China). Thymoma cells were inoculated onto 12-well culture plates prior to transfection, grown to $50 \%$ confluency, and synchronized for $12 \mathrm{~h}$ with serum-free DMEM. Cells were divided
Table I. Polymerase chai reaction primer pair sequences for human Wnt4, JNK and GAPDH.

\begin{tabular}{lc}
\hline Primer & Sequence \\
\hline Wnt4 & \\
Sense & 5'-ACCTGGAAGTCATGGACTCG-3' \\
Antisense & 5'-TCAGAGCATCCTGACCACTG-3' \\
JNK & \\
Sense & 5'-TTTGAGAAACTCTTCCCTGATG-3' \\
Antisense & 5'-ATTGATGTACGGGTGTTGGA-3' \\
GAPDH & \\
Sense & 5'-TGGAGTCTACTGGCGTCTTC-3' \\
Antisense & 5'-TTCACACCCATCACAAACATG-3' \\
\hline
\end{tabular}

JNK, c-Jun N-terminal kinase.

into 6 transfection groups (with 5 experimental repeats), as follows: Control, no interference; TR001, HSHTR001 empty plasmid; Wnt4-shRNA-1, HSH13446-1-CH1 interference plasmid; Wnt4-shRNA-2, HSH13446-2-CH2 interference plasmid; Wnt4-shRNA-3, HSH13446-3-CH3 interference plasmid; and Wnt4-shRNA-4, HSH13446-4-CH4 interference plasmid. Plasmids $(8 \mu \mathrm{g})$ were transfected into each group with Lipofectamine ${ }^{\mathrm{TM}} 2000$ (Invitrogen; Thermo Fisher Scientific, Inc.) for $6 \mathrm{~h}$. Cells were then cultured in DMEM with $10 \% \mathrm{FBS}$ for $48 \mathrm{~h}$ at $37^{\circ} \mathrm{C}$, and total RNA was extracted for PCR using PrimeScript ${ }^{\mathrm{TM}} \mathrm{RT}$ reagent kit and Taq DNA Polymerase (Takara Bio, Inc.). PCR primer pairs for human Wnt4 and GAPDH are listed in Table I. Amplification was performed at $94^{\circ} \mathrm{C}$ for $3 \mathrm{~min}$ for pre-denaturation, followed by 35 cycles of $94^{\circ} \mathrm{C}$ for $30 \mathrm{sec}$ for denaturation, $55^{\circ} \mathrm{C}$ for $30 \mathrm{sec}$ for annealing, and $72^{\circ} \mathrm{C}$ for $30 \mathrm{sec}$ for extension, with a final extension at $72^{\circ} \mathrm{C}$ for $10 \mathrm{~min}$. Wnt4 PCR products were purified by $1.2 \%$ agarose gel. The relative expression of Wnt4 mRNA was calculated with gray values using Quantity One image analysis software (version 4.4; Bio-Rad Laboratories, Inc.) and compared with that prior to transfection in the same cell line (15). Reactions were performed in triplicate. The plasmid exhibiting the best inhibition was selected for subsequent experiments.

Western blotting for Wnt4 and JNK protein expression. Transfected cells were collected in one centrifuge tube for each transfection group and centrifuged at room temperature (110 x g, $5 \mathrm{~min})$. The supernatant was removed, and the cell pellet was washed twice with PBS (0.01 M; pH 7.2-7.3). Each tube of cells was added into $100 \mu \mathrm{l}$ radioimmunoprecipitation assay lysate with phenylmethane sulfonyl fluoride (Sigma-Aldrich; Merck KGaA, Darmstadt, Germany), and protein concentration was determined by bicinchoninic acid assay. Subsequent to quantification and aliquoting, samples were degenerated by boiling. Equal amounts of total protein (30 $\mu \mathrm{g} / \mathrm{sample})$ were subjected to SDS-PAGE (12\%) followed by electrophoretic transfer to nitrocellulose membranes. Nitrocellulose membranes were blocked using 5\% skimmed milk powder for $1 \mathrm{~h}$ at room temperature. Each blot was incubated with 
Table II. Wnt4 short hairpin RNA interference plasmids.

\begin{tabular}{lcccc}
\hline Clone name & Symbol & Location & Length & Target sequence \\
\hline HSH013446-1-CH1(OS241017) & WNT4 & 1475 & 19 & GGTGGAGTAACAAGGAGTA \\
HSH013446-2-CH1(OS241018) & WNT4 & 1845 & 19 & GAAGAGGAAACTTAACCAC \\
HSH013446-3-CH1(OS241019) & WNT4 & 2289 & 19 & GCAGACAAACCAAGAATGC \\
HSH013446-4-CH1(OS241020) & WNT4 & 2973 & 19 & AACGTCCGAGATTCGGAAT
\end{tabular}

primary antibodies overnight at $4^{\circ} \mathrm{C}$ [rabbit anti-human Wnt4 polyclonal antibody (dilution 1:100, cat. no. ab94742; Abcam), mouse anti-human JNK polyclonal antibody (dilution 1:500, cat. no. ab201624; Abcam) and rabbit antihuman $\beta$-actin polyclonal antibody (dilution 1:1,000, cat. no. bs-0061R; Bioss, Beijing, China)] and a secondary antibody for $60 \mathrm{~min}$ at room temperature [goat anti-rabbit/rat monoclonal antibodies (dilution 1:8,000, cat. nos. SA00001-1 and SA00001-2, respectively; ProteinTech Group, Inc., Chicago, IL, USA)]. Nitrocellulose membranes were washed with enhanced chemiluminescence developing solution (K-12045-D20; Advansta, Inc., Menlo Park, CA, USA), exposed and developed using X-ray film. Grey scale analysis was performed using Quantity One software (Bio-Rad Laboratories, Inc.).

Detection of apoptosis. Thymoma cells were divided into 4 groups, as follows: Empty control, Lipofectamine 2000, TR001 plasmid, and Wnt4-shRNA-3 plasmid. The aforementioned transfection steps were used, and cell proliferation was observed using a Leica DMIL microscope (Leica Microsystems $\mathrm{GmbH}$; magnification, x100) after $48 \mathrm{~h}$. When cells were $80-90 \%$ confluent and in good condition, culture medium (DMEM) was removed and cells were gently rinsed twice with 1X PBS. Wright-Giemsa and Hoechst-33342/propidium iodide (PI) (both from Beijing Solarbio Science and Technology Co., Ltd., Beijing, China) fluorescent double staining was used to stain cells. Apoptosis was quantified by flow cytometry.

Wright-Giemsa double staining. Ethanol (1 ml; 95\%) was added to each culture well and cells were fixed for $15 \mathrm{~min}$. The ethanol was removed and $1 \mathrm{ml}$ Wright-Giemsa staining solution was added. The cells were stained for $2 \mathrm{~min}$ at room temperature. The staining solution was discarded, the cells were rinsed twice in flowing water and observed using a Leica DMIL microscope (magnification, $x 400$ ), and images were captured. Apoptotic cells exhibited chromatin condensation, nuclear membrane fragmentation, and appearance of apoptotic bodies. Light staining was also observed in necrotic cells.

Hoechst-33342/PI fluorescent double staining. Damping fluid (2 ml), Hoechst 33342 staining solution $(10 \mu \mathrm{l})$ and PI staining solution $(10 \mu \mathrm{l})$ (Solarbio Science and Technology Company, Ltd.) were added to each well and mixed lightly. Cells were kept in the dark for $20 \mathrm{~min}$ at $4^{\circ} \mathrm{C}$. The staining solution was then discarded and the cells were rinsed with $1 \mathrm{X}$ PBS. Physiological saline $(1 \mathrm{ml})$ was added, and fluorescent images were captured using a Leica DMIL microscope (magnification, x200). Normal cells stained blue and the nucleolus

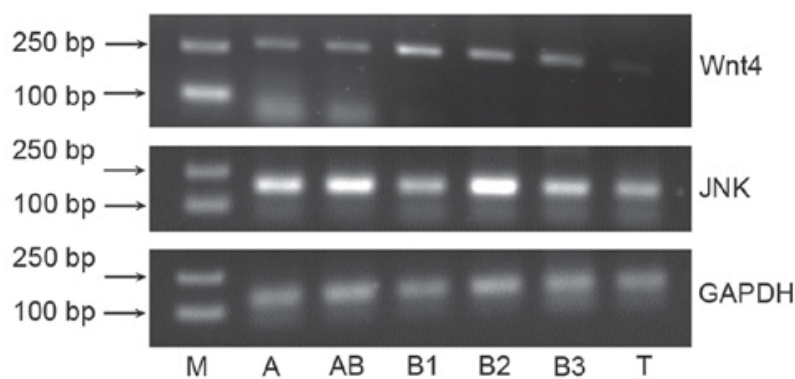

Figure 1. Agarose gel electropherogram of Wnt4, JNK and GAPDH mRNA expression. Lane M, marker; lane A, type A thymoma; lane AB, type AB thymoma; lane B1, type B1 thymoma; lane B2, type B2 thymoma; lane B3, type B3 thymoma; and lane T, thymus. JNK, c-Jun N-terminal kinase.

structure was normal. Apoptotic cells stained bright blue and light red, and necrotic cells stained light blue and bright red.

Annexin V-fluorescein isothiocyanate (FITC)/PI double staining and flow cytometry. Apoptosis was detected by flow cytometry with Annexin V-FITC/PI kit (cat. no. FXP018-050; 4A Biotech Co., Ltd., Beijing, China), which distinguishes between apoptotic and dead cells based on differential membrane staining. Different cells can be distinguished from the four quadrant diagram: Normal live cells are Annexin $\mathrm{V}^{-} / \mathrm{PI}^{-}$ cells (Q3); viable apoptotic cells are Annexin $\mathrm{V}^{+} / \mathrm{PI}^{-}$cells (Q4); and advanced apoptotic cells and dead cells are Annexin $\mathrm{V}^{+} / \mathrm{PI}^{+}$ cells (Q2). The percentage of apoptosis was determined by statistical analysis of Q4 data. Fluorescence was measured with a flow cytometer (FACSCanto II, BD Biosciences, Franklin Lakes, NJ, USA) and analyzed using FACSDiva version 6.1.3 (BD Biosciences).

Statistical analysis. Statistical analysis was conducted using the t-test for paired samples, one-way analysis of variance for multi group data, multiple comparisons between groups were performed using least significant difference method, and Fisher's exact test for categorical data in $2 \times 2$ contingency tables. $\mathrm{P}<0.05$ was considered to indicate a statistically significant difference. Data were processed using SPSS 19.0 (IBM Corp., Armonk, NY, USA).

\section{Results}

Expression of Wnt4 and JNK $m R N A$ and protein in thymoma. Wnt4 and JNK mRNA expression was detected in thymoma tissues (Fig. 1). The relative expression of Wnt4 mRNA was $4.45 \pm 1.79(\mathrm{P}<0.001)$, and JNK mRNA was $2.61 \pm 0.83$, compared with normal thymus tissues (Fig. 2; $\mathrm{P}<0.001$ ). 


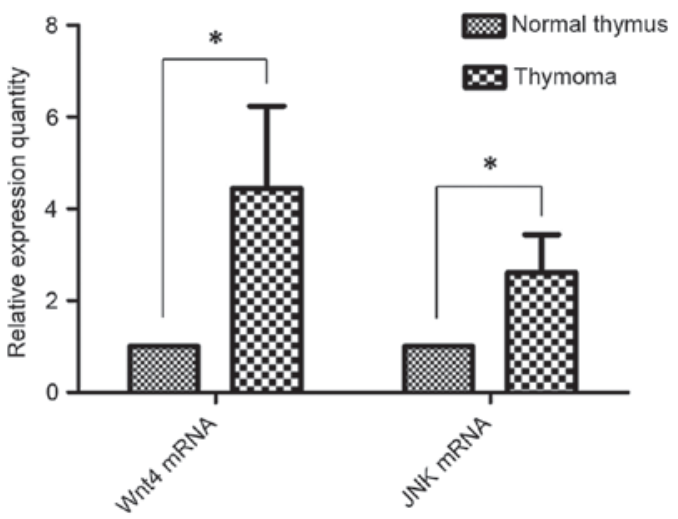

Figure 2. Relative expression of Wnt4 and JNK mRNA in normal thymus and thymoma tissues. Relative expression of Wnt 4 and JNK mRNA in thymoma tissue (4.45 \pm 1.79 and $2.61 \pm 0.83$, respectively) was elevated compared with normal thymus tissue. ${ }^{*} \mathrm{P}<0.001$. JNK, c-Jun $\mathrm{N}$-terminal kinase.

The positive staining of Wnt4 protein in normal thymus and thymoma tissues was 0.0 and $93.3 \%$, respectively $(\mathrm{P}<0.001)$, and positive staining of $\mathrm{JNK}$ protein in normal thymus tissue and thymoma was 16.7 and $80.0 \%$, respectively $(\mathrm{P}=0.014)$ (Table III; Fig. 3).

Screening of psi-H1-shRNA-Wnt4 interference plasmid and its inhibitory effect on Wnt4. The plasmids of positive electrophoresis strips were selected and sent to sequence analysis (Aoke Biotechnology Co., Ltd.). Results showed that the complete target gene inserted, and that the sequence was identical with the sequence designed.

Wnt4 expression was determined by semi-quantitative RT-PCR (Fig. 4). The results showed that Wnt4 expression in each experimental group was decreased compared with the controls. However, Wnt4-1 expression in the shRNA-group was elevated compared with the TR001 group, indicating that this plasmid did not successfully inhibit expression. Expression in the other experimental groups was lower than in the TR001 group, indicating that the shRNA-Wnt4 interference plasmid was constructed successfully, and effectively inhibits Wnt4 expression. The relative inhibitions of the four candidate plasmids were $15.50,40.87,52.37$ and $34.89 \%$ respectively. Expression following transfection of shRNA-Wnt4-3 was the lowest $(\mathrm{P}<0.01)$. Therefore, shRNA-Wnt4-3 was selected as the best silencing plasmid and used in subsequent experiments.

Wht4 shRNA inhibits JNK. JNK expression decreased after Wnt4 shRNA, as detected by semi-quantitative RT-PCR, consistent with Wnt4 expression level $(\mathrm{P}<0.01)$, indicating that transfection of shRNA-Wnt4-3 inhibits JNK expression (Fig. 5A and B).

Western blot analysis demonstrated JNK and Wnt4 protein expression in the control and TR001 groups was significantly increased compared with the shRNA-Wnt4-3 group $(\mathrm{P}<0.05)$, although $\beta$-actin levels had not changed (Fig. 5C). This indicated that the shRNA-Wnt4 plasmid inhibits Wnt4 protein expression, which suppresses JNK protein expression.

Detection of apoptosis subsequent to transfection WrightGiemsa staining. The cellular morphology of control and
Table III. Cases of positive expression of Wnt4 and JNK protein in normal thymus tissue and thymoma.

\begin{tabular}{lrrrrrrr}
\hline & \multicolumn{2}{c}{ Wnt4 expression, $n$} & & \multicolumn{2}{c}{ JNK expression, $\mathrm{n}$} \\
\cline { 2 - 4 } \cline { 6 - 8 } Tissue & + & - & P-value & & + & - & P-value \\
\hline Thymus & 0 & 6 & 0.000 & & 5 & 0.014 \\
Thymoma & 14 & 1 & & 12 & 3 & \\
\hline
\end{tabular}

JNK, c-Jun N-terminal kinase.

Lipofectamine 2000 groups was polygonal. The cytoplasmic staining was light and uniform, with hyperchromatic nuclei. Nuclear morphology indicated the cellular division phase, but no apoptotic bodies or cytoplasmic vacuoles were observed. These observations indicated that thymomas in the control and Lipofectamine 2000 groups were not undergoing apoptosis. However, characteristic apoptotic morphological changes, such as nuclear chromatin agglutination, invagination of the cellular membrane, and apoptotic body formation were observed in the TR001 and shRNA transfection groups. These morphological changes were more prevalent in the shRNA-transfected groups (Fig. 6).

Hoechst-33342/PI fluorescent double staining. Normal cells stain low bright blue and no red (Hoechst $33342^{+} / \mathrm{PI}^{-}$), with control cells in the visual field having a typical polygon shape. In the Lipofectamine 2000 group, a small number of bright blue (Hoechst $33342^{++} / \mathrm{PI}^{-}$) cells were normal or undergoing early stage apoptosis, but there were no red-stained cells. Transfection of plasmid TR001 resulted in a small number of blue and red double stained apoptotic cells (Hoechst 33342 ${ }^{++} / \mathrm{PI}^{+}$). Notably, shRNA transfection produced a large number of apoptotic (Hoechst $33342^{++} / \mathrm{PI}^{+}$) and necrotic (Hoechst 33342 $/ \mathrm{PI}^{++}$) cells (Fig. 7).

Flow cytometry. Apoptosis in the Lipofectamine 2000 and TR001 groups was $11.07 \pm 0.35 \%$ and $11.17 \pm 0.35 \%$, respectively, although they did not differ compared with controls $(10.40 \pm 0.56 \%)(\mathrm{P}>0.05)$. The apoptosis rate following shRNA transfection was $14.70 \pm 0.62 \%$, which was significant compared with controls (Fig. 8; $\mathrm{P}<0.001$ ).

\section{Discussion}

Few studies have investigated thymoma signaling pathways. Notably, Wnt receptors are present in all thymocyte subsets (16), and Wnt pathways are associated with malignant tumors. Wnt-4 activates the JNK-dependent noncanonical signaling pathway (17). Ma et al (18) demonstrated that the overexpression of c-Jun in thymus epithelial tumors was closely associated with the pathogenesis and biological behavior of the neoplasm (18). Thus, the present study aimed to explore the role that Wnt4 signaling may play in promoting thymoma cell proliferation via a non-classical JNK-mediated pathway. The present results showed that the thymoma Wnt4 and JNK gene and protein expression 


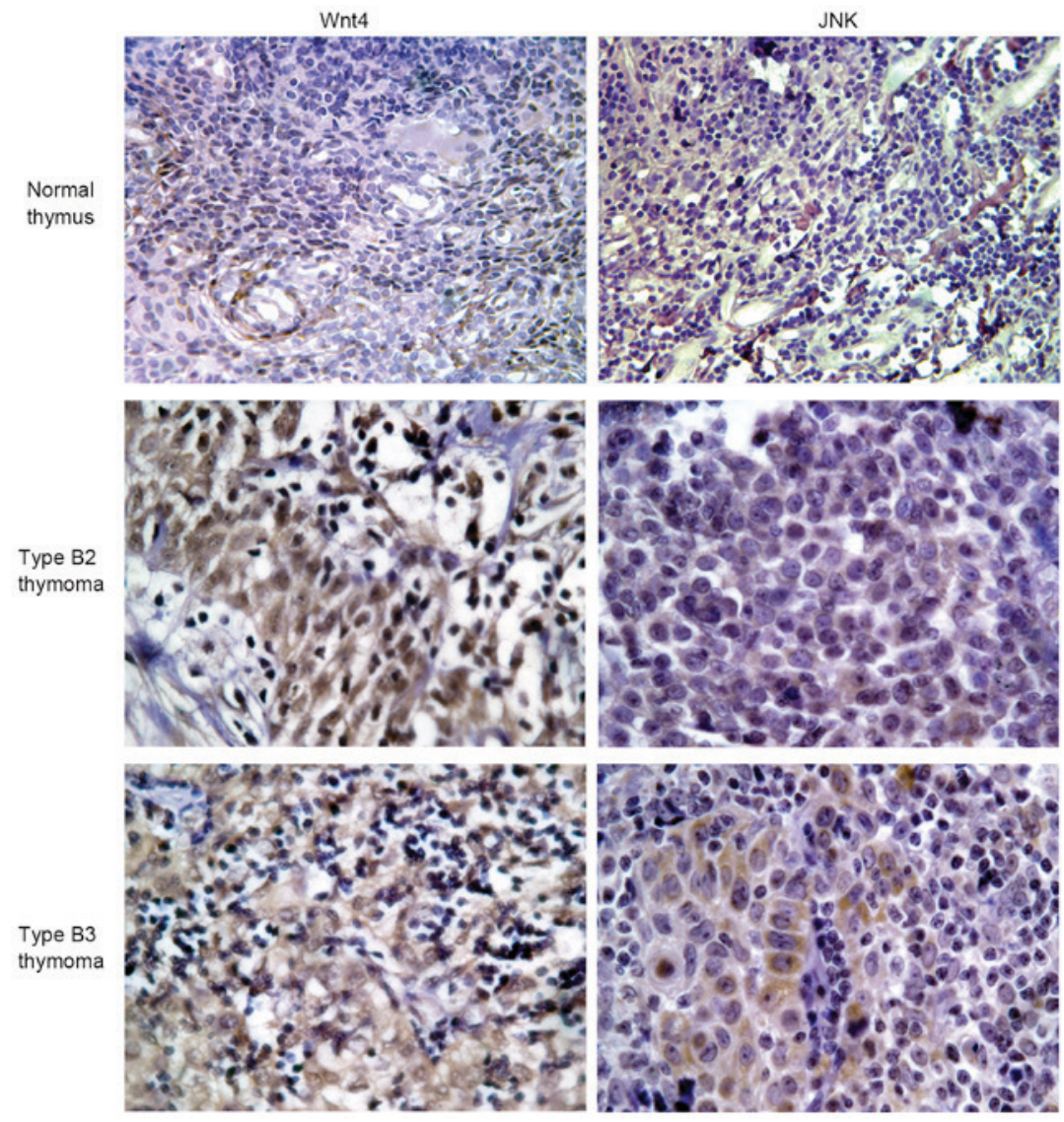

Figure 3. Immunohistochemistry of Wnt4 and JNK in thymus and thymoma, at x400 magnification. Wnt4-positive granules stain brown in the cell membrane and cytoplasm of normal thymus, type B2 thymoma and type B3 thymoma (left three images). JNK-positive granules stained pale brown in the cytoplasm of normal thymus, type B2 thymoma and type B3 thymoma (right three pictures). JNK, c-Jun N-terminal kinase.

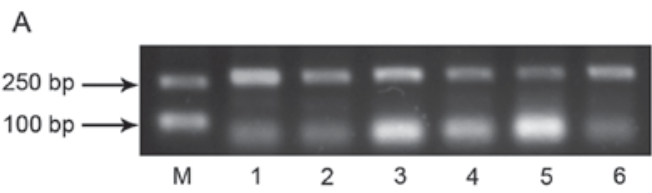

B

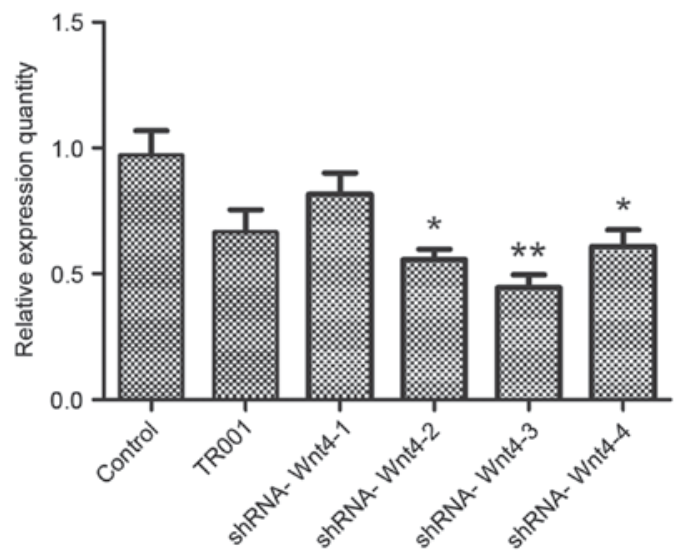

Figure 4. Wnt4 mRNA expression following candidate plasmid transfection (A) Agarose gel electropherogram of Wnt4 mRNA expression following transfection. (B) Evaluation of mRNA expression levels showed that plasmid shRNA-Wnt4 effectively silences Wnt4 expression in the latter three groups, but shRNA-Wnt4-3 was most effective. Therefore, shRNA-Wnt4-3 was selected as the best interference plasmid and used in subsequent experiments. " $\mathrm{P}<0.05$ and ${ }^{* *} \mathrm{P}<0.01$ vs. control group. shRNA, short hairpin RNA; Lane M, marker; lane 1, control; lane 2, TR001; lane 3, shRNA-Wnt4-1; lane 4, shRNA-Wnt4-2; lane 5, shRNA-Wnt4-3; and lane 6, shRNA-Wnt4-4. was elevated relative to that in the normal thymus. In addition, Wnt4 and JNK expression and apoptosis changes were observed in thymoma cells subsequent to Wnt4 gene silencing.

Since Napoli et al (19) identified botanic co-suppression in 1990, RNAi technology has been widely used. In the present study, the Wnt4 shRNA sequence was designed and a shRNA plasmid vector was constructed to silence Wnt4. Sequence analysis showed that Wnt4 shRNA was successfully inserted into the expression vector and effectively inhibited Wnt4 expression, indicating that the interference plasmid was constructed successfully. The shRNA-Wnt4-3 inhibition was $52.37 \%$, which was the best of four constructed plasmids $(\mathrm{P}<0.01)$, prompting us to chose this plasmid for subsequent experiments. Although certain studies have shown that the development of thymus epithelial cells requires activation of the $\beta$-catenin-mediated canonical Wnt signaling pathway $(11,20,21)$, elevated expression of $\beta$-catenin was not observed in the present study. However, expression of the JNK mRNA and protein were significantly decreased following transfection of the shRNA-Wnt4 plasmid compared with the controls. This result may indicate that the Wnt4-activated pathway is a JNK-mediated PCP-like pathway in thymoma.

Cancer cells escape apoptosis by manipulating the expression level of anti-apoptotic molecules or inactivating apoptotic elements (22). Studies have shown that inhibition of Wnt 
A

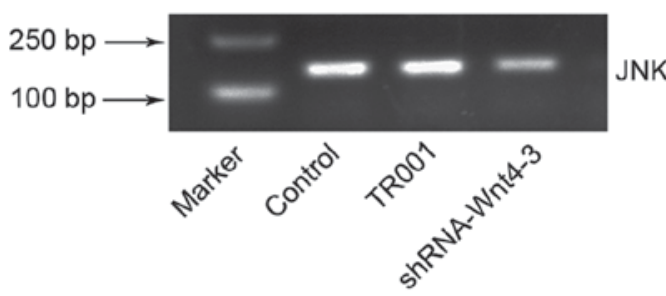

C

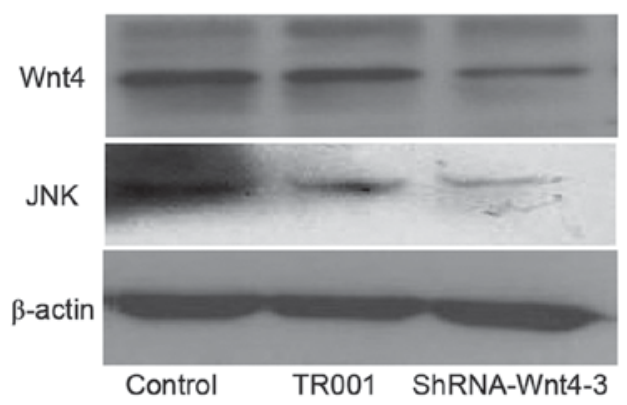

B

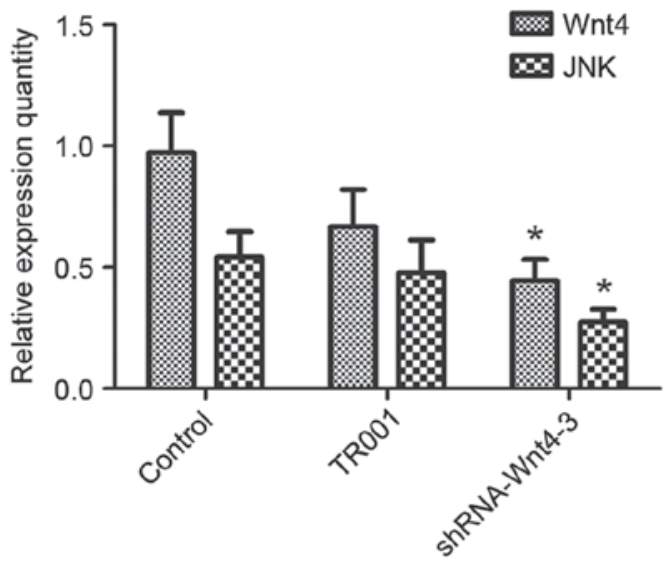

Figure 5. Wnt 4 and JNK mRNA and protein expression subsequent to shRNA-Wnt4-3 transfection. These results indicated that the shRNA-Wnt4 plasmid inhibits JNK mRNA expression, which suppresses Wnt4 and JNK protein expression. (A) Agarose gel electropherogram of JNK mRNA after Wnt4 shRNA. (B) Relative expression of Wnt4 and JNK mRNA subsequent to plasmid transfection. " $\mathrm{P}<0.01$ vs. control group. (C) Wnt4 and JNK protein expression. JNK, c-Jun N-terminal kinase.
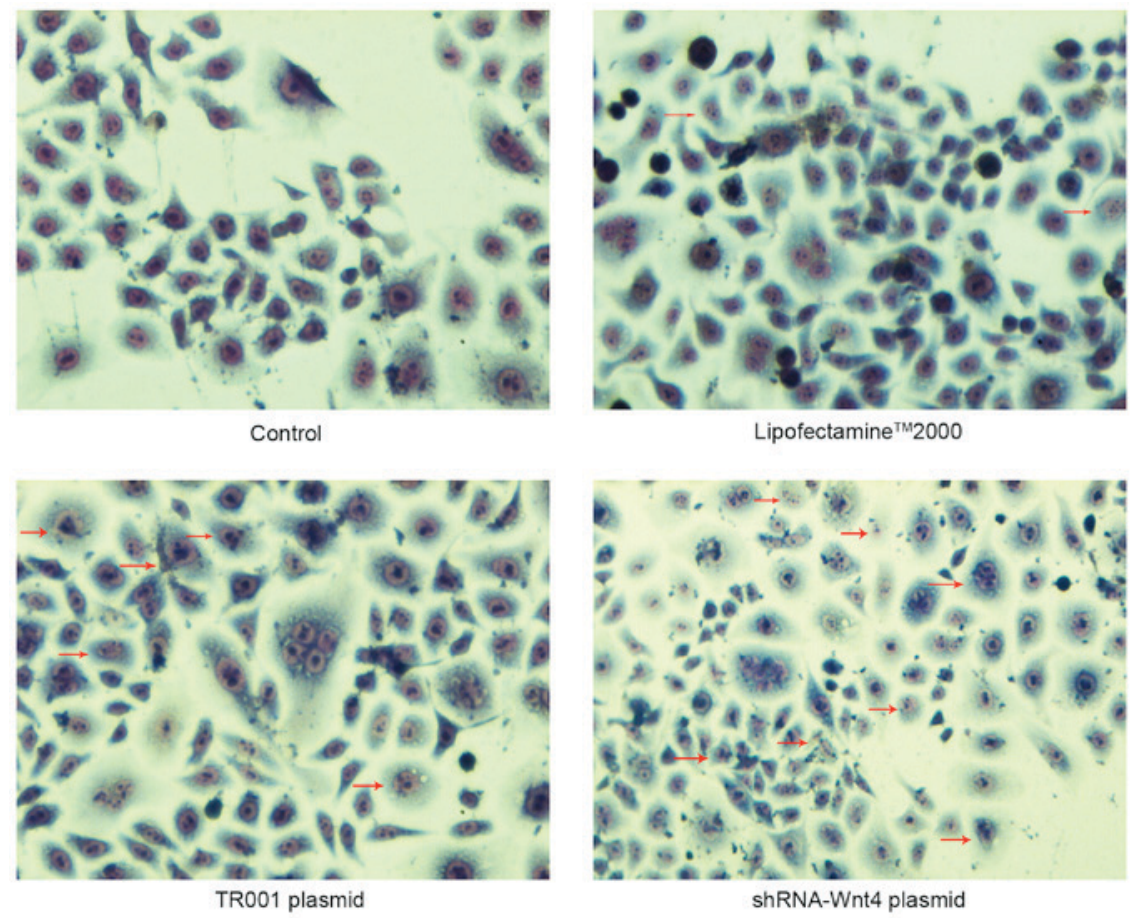

Figure 6. Wright-Giemsa staining in each group at $48 \mathrm{~h}$ post-transfection. Red arrows indicate apoptotic cells at x400 magnification.

signaling induces apoptosis and suppresses cell proliferation $(23,24)$. Age associated changes in thymus cell number and function were accompanied by a decrease in the transcription levels of Wnt4, and activation of the Wnt4 signaling pathway promoted thymus epithelial cell proliferation and alleviated apoptosis (25). Therefore, the present study qualitatively (Wright-Giemsa and Hoechst-33342/PI staining) and quantitatively (Annexin V-FITC/PI staining) analyzed thymoma apoptosis subsequent to downregulation of Wnt4 by transfection with the shRNA-Wnt4-3 plasmid. The results indicated that cells exhibited increased cellular apoptosis and characteristic apoptotic morphological changes, including nuclear chromatin agglutination, and apoptotic body formation, compared with controls. Collectively, these results 


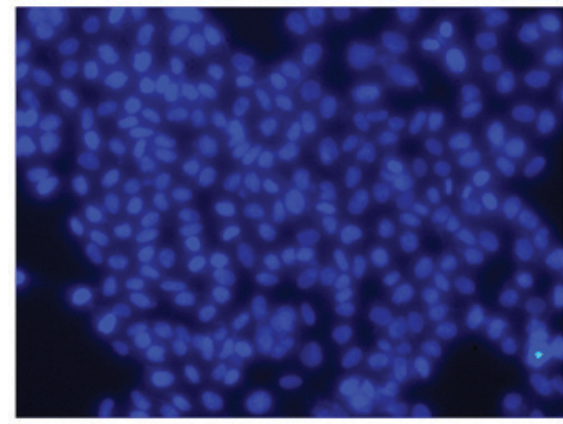

Control

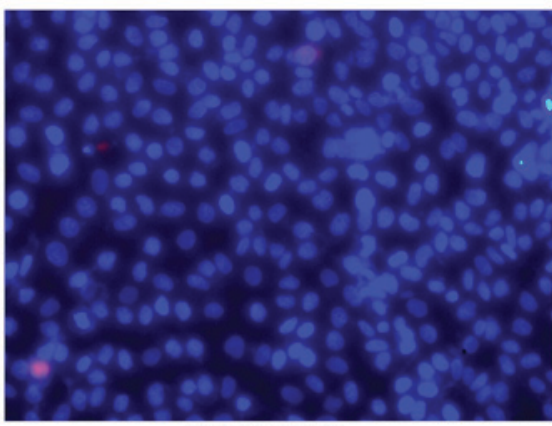

TR001 plasmid

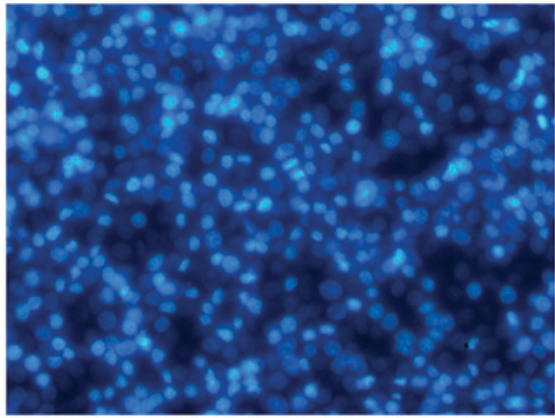

Lipofectamine $^{T M} 2000$

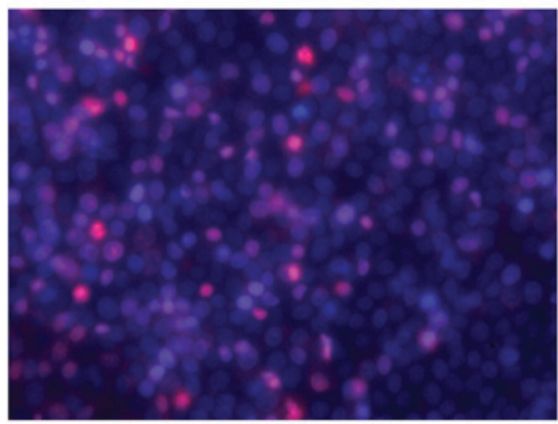

shRNA-Wnt4 plasmid

Figure 7. Hoechst-33342/propidium iodide staining in each transfected group after $48 \mathrm{~h}$ at $\times 200$ magnification.

A

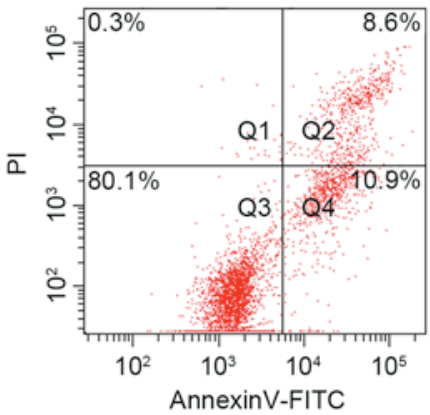

C

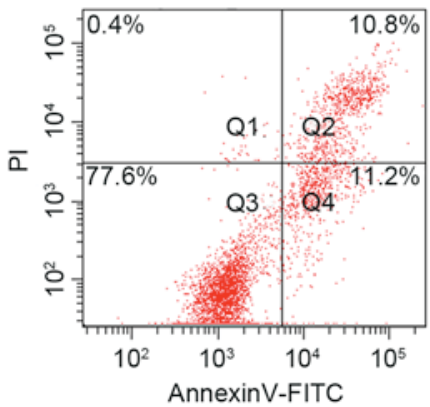

B

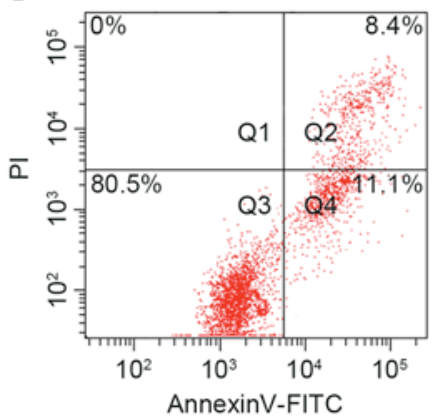

D

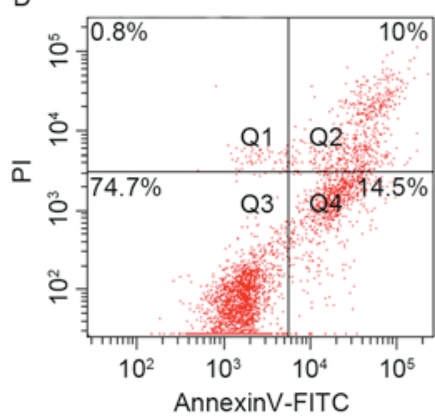

E

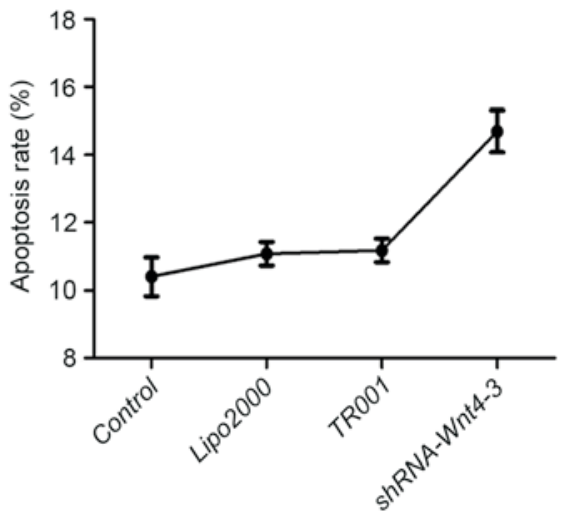

Figure 8. Flow cytometry analysis of cellular apoptosis subsequent to transfection in the (A) control, (B) Lipofectamine ${ }^{\mathrm{TM}} 2000$, (C) TR001 and (D) shRNA-Wnt4-3 groups. (E) Apoptosis rate in each group. Cells in Q2 are undergoing advanced apoptosis, cells in Q3 are normal live cells and cells in Q4 are viable apoptotic cells.

indicate that downregulation of Wnt4 promotes thymoma apoptosis, and that Wnt4 activation may be one of the causes of thymoma development.

The present results suggest that thymomas exhibit elevated Wnt4 and JNK mRNA and protein expression. Thymomas also show increased apoptosis subsequent to RNAi inhibition of Wnt4. Thus, Wnt4 may have an important role in promoting thymoma development, and may be activated through a JNK mediated PCP-like pathway.

\section{Acknowledgements}

This study was supported by the Natural Sciences Foundation of Tianjin (grant no. 14JCZDJC57100) and the Special Fund 
for Clinical Research of Wu Jieping Medical Foundation (grant no. 320.6750.14318). English language editing was provided by Edanz China (Beijing, China).

\section{References}

1. Singh G, Rumende CM and Amin Z: Thymoma: Diagnosis and treatment. Acta Med Indones 43: 74-78, 2011.

2. Detterbeck FC: Evaluation and treatment of stage I and II thymoma. J Thorac Oncol 5 (Suppl 4): S318-S322, 2010.

3. Marx A, Willcox N, Leite MI, Chuang WY, Schalke B, Nix W and Ströbel P: Thymoma and paraneoplastic myasthenia gravis. Autoimmunity 43: 413-27, 2010.

4. Peifer M and Polakis P: Wnt signaling in oncogenesis and embryogenesis - a look outside the nucleus. Science 287: 1606-1609, 2000.

5. Huang C, Ma R, Xu Y, Li N, Li Z, Yue J, Li H, Guo Y and Qi D: Wnt2 promotes non-small cell lung cancer progression by activating WNT/ $\beta$-catenin pathway. Am J Cancer Res 5: 1032-1046, 2015.

6. Chiurillo MA: Role of the Wnt/ $\beta$-catenin pathway in gastric cancer: An in-depth literature review. World J Exp Med 5: 84-102, 2015.

7. MacMillan CD, Leong HS, Dales DW, Robertson AE, Lewis JD, Chambers AF and Tuck AB: Stage of breast cancer progression influences cellular response to activation of the WNT/planar cell polarity pathway. Sci Rep 4: 6315, 2014.

8. van Loosdregt J, Fleskens V, Tiemessen MM, Mokry M, van Boxtel R, Meerding J, Pals CE, Kurek D, Baert MR, Delemarre EM, et al: Canonical Wnt signaling negatively modulates regulatory T cell function. Immunity 39: 298-310, 2013.

9. Kvell K, Varecza Z, Bartis D, Hesse S, Parnell S, Anderson G, Jenkinson EJ and Pongracz JE: Wnt4 and LAP2alpha as pacemakers of thymic epithelial senescence. PLoS One 5: e10701,2010.

10. Heinonen KM, Vanegas JR, Brochu S, Shan J, Vainio SJ and Perreault C: Wnt4 regulates thymic cellularity through the expansion of thymic epithelial cells and early thymic progenitors. Blood 118: 5163-5173, 2011.

11. Kvell K, Fejes AV, Parnell SM and Pongracz JE: Active Wnt/beta-catenin signaling is required for embryonic thymic epithelial development and functionality ex vivo. Immunobiology 219: 644-652, 2014.

12. Tejada-Romero B, Carter JM, Mihaylova Y, Neumann B and Aboobaker AA: JNK signalling is necessary for a Wnt- and stem cell-dependent regeneration programme. Development 142: 2413-2424, 2015.

13. Livak KJ and Schmittgen TD: Analysis of relative gene expression data using real-time quantitative PCR and the 2(-Delta Delta C(T)) method. Methods 25: 402-408, 2001
14. Wang G, Wang Y, Zhang P, Chen Y, Liu Y, Guo F and Zhang H: Establishment and characterization of a novel cell line derived from thymoma with myasthenia gravis patients. Thoracic Cancer 6: 194-201, 2015.

15. Kojima S and Borisy GG: An image-based, dual fluorescence reporter assay to evaluate the efficacy of shRNA for gene silencing at the single-cell level. F1000Res 3: 60, 2014.

16. Weerkamp F, Baert MR, Naber BA, Koster EE, de Haas EF, Atkuri KR, van Dongen JJ, Herzenberg LA and Staal FJ: Wnt signaling in the thymus is regulated by differential expression of intracellular signaling molecules. Proc Natl Acad Sci USA 103: 3322-3326, 2006.

17. Heinonen KM, Vanegas JR, Lew D, Krosl J and Perreault C: Wnt4 enhances murine hematopoietic progenitor cell expansion through a planar cell polarity-like pathway. PLoS One 6: e19279, 2011.

18. Ma Y, Li Q, Cui W, Miao N, Liu X, Zhang W, Zhang C and Wang J: Expression of c-Jun, p73, Casp9, and N-ras in thymic epithelial tumors: Relationship with the current WHO classification systems. Diagn Pathol 7: 120, 2012.

19. Napoli C, Lemieux $\mathrm{C}$ and Jorgensen R: Introduction of a chimeric chalcone synthase gene into petunia results in reversible co-suppression of homologous genes in trans. Plant Cell 2: 279-289. 1990.

20. Liang CC, You LR, Yen JJ, Liao NS, Yang-Yen HF and Chen CM: Thymic epithelial $\beta$-catenin is required for adult thymic homeostasis and function. Immunol Cell Biol 91: 511-523, 2013.

21. Zuklys S, Gill J, Keller MP, Hauri-Hohl M, Zhanybekova S, Balciunaite G, Na KJ, Jeker LT, Hafen K, Tsukamoto N, et al: Stabilized beta-catenin in thymic epithelial cells blocks thymus development and function. J Immunol 182: 2997-3007, 2009.

22. Igney FH and Krammer PH: Death and anti-death: Tumour resistance to apoptosis. Nat Rev Cancer 2: 277-288, 2002.

23. Zhang KS, Zhou Q, Wang YF and Liang LJ: Inhibition of Wnt signaling induces cell apoptosis and suppresses cell proliferation in cholangiocarcinoma cells. Oncol Rep 30: 1430-1438, 2013.

24. Bilir B, Kucuk O and Moreno CS: Wnt signaling blockage inhibits cell proliferation and migration, and induces apoptosis in triple-negative breast cancer cells. J Transl Med 11: 280, 2013.

25. Wei T, Zhang N, Guo Z, Chi F, Song Y and Zhu X: Wnt4 signaling is associated with the decrease of proliferation and increase of apoptosis during age-related thymic involution. Mol Med Rep 12: 7568-76, 2015.

This work is licensed under a Creative Commons Attribution-NonCommercial-NoDerivatives 4.0 International (CC BY-NC-ND 4.0) License. 